\title{
Alumnas de nivel bachillerato de la ESTi: Roles durante el periodo de pandemia
}

\author{
ESTi high school students: Roles during the pandemic period \\ Mayra Anaid Valerio Nolasco ${ }^{a}$, Gaby Yolanda Vega Cano ${ }^{b}$ Sandra Zapata Salinas ${ }^{c}$ Cristina \\ Flores Amador ${ }^{a}$
}

\begin{abstract}
:
The purpose of this document is to make visible the roles that female students have adopted during the pandemic period, since a year ago the UAEH took classes at home as a preventive measure, from the first moment with B-learning modality and later totally virtual. These students have had to adapt to academic work, at home and even take care of sick relatives or in academic support to min ors. The pandemic period took all institutions by surprise: the family, the school, the private sector, the health sector, in general each of the inhabitants. Marcela Lagarde's theory of the captivity of women is taken up to identify them in these female students.
\end{abstract}

Keywords:

Roles, pandemic, school, family

\section{Resumen:}

El presente escrito tiene la finalidad de visibilizar los roles que han adoptado las alumnas durante el periodo de pandemia, desde hace un año que la UAEH tomó como medida preventiva tomar clases en casa. Dichas alumnas han tenido que adaptarse al trabajo académico, en casa e incluso cuidar a familiares enfermos o en apoyo académico a menores de edad. El periodo de pandemia tomó por sorpresa a todas las instituciones: la familia, la escuela, el sector privado, el sector salud, en general a cada uno de los habitantes. Se retoma la teoría de Marcela Lagarde de los cautiverios de las mujeres, para identificarlos en dichas alumnas.

\section{Palabras Clave:}

Roles, pandemia, escuela, familia

\section{Introducción}

Según la OMS, una "pandemia" se declara cuando se expande de manera mundial una enfermedad (OMS, 2010). Generalmente las enfermedades que se han convertido en pandemia son las relacionadas con gripes, que afectan en mayor manera a los adultos mayores. Los adultos mayores han sido los más afectados por la pandemia COVID-19 en las américas, según
Organización Panamericana de la Salud, afectando no solo su salud sino su economía en general, impidiéndole realizar sus actividades de manera habitual y para lograr tener un mayor cuidado de ellos y la población en general se realizaron medidas extraordinarias a nivel mundial, como es, tomar clases desde casa a nivel nacional.

La UAEH se declara en suspensión de clases por pandemia a partir del 21 de marzo del 2021 y se menciona

a Mayra Anaid Valerio Nolasco, Universidad Autónoma del Estado de Hidalgo, https://orcid.org/0000-0003-2155-1250, Email: mayra_valerio@uaeh.edu.mx

b Gaby Yolanda Vega Cano, Universidad Autónoma del Estado de Hidalgo, https://orcid.org/0000-0003-1440-1617, Email: gaby@uaeh.edu.mx

Sandra Zapata Salinas, Universidad Autónoma del Estado de Hidalgo, https://orcid.org/0000-0001-9934-9605, Email: szapata@uaeh.edu.mx

d Cristina Flores Amador, Universidad Autónoma del Estado de Hidalgo, http://orcid.org/0000-0001-8122-3094, Email: floresa@uaeh.edu.mx 
que el regreso será el lunes 20 de abril (UAEH, 2020) . Para abril del 2021, ya se cumplió un año que se declaró esta emergencia sanitaria en la UAEH y aun no se determina la fecha del posible regreso a clases.

La comunidad estudiantil de la ESTi y en general de toda la UAEH se ha tenido que adaptar para continuar con las actividades académicas y de enseñanza aprendizaje.

\section{¿Por qué estudiar a las mujeres?}

Mujer es una categoría general para referirse al género femenino y a su condición histórica, de ser denominadas un ser social genérico (Lagarde, 2003), en esta sociedad denominada patriarcal, la mujer se define por su sexualidad, por el trabajo y por la naturaleza, la mujer como tal no tiene existencia material, es una categoría producto de la abstracción de un conjunto de características que comparten todas las mujeres.

Cada cultura ha desarrollado un concepto de mujer que se ha construido socialmente a base de las necesidades de los demás, por ello en cada cultura la mujer tiene un concepto, un papel, un rol y sobre todo un trabajo que debe desempeñarse en y para la sociedad. Cada cultura denomina y construye el concepto de mujer de manera distinta.

"Las mujeres" es la categoría que se expresa a las particulares para ubicar la situación histórica de cada una de ellas y estas, en particular se determinan por un conjunto de relaciones sociales como las genéricas, de clase edad, escolaridad, condición, religión, trabajo, salud, entre otros (Lagarde, 2003).

Lagarde en el 2003, realizó un estudio antropológico de las mujeres, y designo categorías de análisis para describir su situación, cada una expresa las situaciones de poder a las que son sometidas socialmente.

Los roles para esta investigación son: "Son comportamientos, tareas y funciones que una persona debe desempeñar según su sexo, y que, por estar construidos social y culturalmente, pueden cambiar. En todas las sociedades las tareas que realizan los hombres gozan de mayor prestigio social que las realizadas por las mujeres" (CNDH, 2021), se clarifica esto con la finalidad de que el lector tenga una mayor comprensión.

\section{Madreesposas}

Esta categoría encasilla a las mujeres por dos actividades: ser madre y ser esposa, desde antes de nacer las mujeres ya están destinadas socialmente a cumplir ese rol. Por ello la mujeres tienen que vivir de acuerdo con las normas establecidas socialmente por una parte ligados a los hijos y por otra a los esposos, siendo esta última no necesariamente a los roles conyugales sino también a los cuidados de los padres, suegros, tíos, entre otros.
Esta categoría se desarrolla a partir de las relaciones, las funciones y el poder, que permite la intercambiabilidad de los sujetos y su posibilidad dual de ser madres y esposas de acuerdo a las circunstancias (Lagarde, 2003); esta categoría va permeada por el poder y los sujetos que en este caso son las madresesposas, que siempre van ligadas al cuidado y al servicio de los otros; ser una madre es sinónimo de sacrificio y entrega, ser una esposa es sinónimo de abandonar su vida de soltera donde solo pensaba en sí misma para ahora entregarle su vida a su esposo y vivir para él.

Según Lagarde, el poder y sus varias manifestaciones se observan en esta categoría de análisis de la mujer, por un lado, se define antropológicamente el papel de la mujer como madre, o como esposa, que no necesariamente se debe tener un esposo o hijos para cumplir con este rol; siendo la conyugalidad y la maternidad, ejes socioculturales y políticos que definen las condición en general de las mujeres (Lagarde, 2003).

Como ya se mencionó no se necesita tener hijos para cumplir el rol de madre ya que se puede ser madre de un amigo, de una hermano o de un novio, cualquier persona que esté cerca de la mujer y sea adoptado por ella para cuidarlo, protegerlo, estar al pendiente en su vida, y cumplir todas las actividades de cuidado que se deben atender como madre, por ello según Largarde, la mujer maternaliza de manera simbólica, económica, social imaginaria y afectiva (Lagarde, 2003).

Por otra parte, tampoco la conyugalidad tiene que cumplir el requisito del matrimonio o vivir en la misma casa con una pareja sentimental; se puede ser esposo de cualquier persona con que las mujeres se relacionan, no precisamente se debe relacionarse de la forma sexual sino también de las antes mencionadas, por ejemplo cuando las mujeres se toman el papel de cuidar a sus padres viudos y los atienden, o cuando sus hermanos se separan de las esposas, del mismo modo las mujeres asumen un rol de esposas sin asumir el erotismo conyugal.

De esta forma se considera a esta categoría como ejes socioculturales y políticos (Lagarde, 2003), que definen la condición general de las mujeres en la vida y en la cultura. El poder se observa en esta categoría en el momento en que una mujer se desprende de su vida para complacer a otros, desde la perspectiva de (Foucault, 1988) este tipo de poder es el pastoral, donde adopta las necesidades de los demás y lo interioriza para dejar ser ella misma e intentar satisfacer la necesidades de ella; en este punto las mujeres dejan de ser mujeres y se convierten en "seres para los otros". Esta relación de poder se manifiesta en todas aquellas mujeres que nacen en determinada cultura y se enrolan desde su nacimiento en cualquiera de las dos actividades (Foucault, 1988). 
El poder se manifiesta desde lo simbólico en un primer momento cuando una mujer se casa, "pierde su libertad" y se convierte en la ama de su casa que está al servicio de los demás: su esposo, su familia, sus suegra, entre otros, porque se dedica a agradar a toda la familia, incluso abandonando sus propios gustos, porque ahora es una mujer casada y debe comportarse de una manera diferente; en un segundo momento cuando las mujeres tienen hijos, cumplen una doble función de cuidar y proteger a sus hijos y esposo pero al mismo tiempo también agradar a la familia en la forma de cuidarlos, educarlos, vestirlo, protegerlos, siempre se dedica a satisfacer las necesidades de los demás antes de las propias, tiene que ser una mujer para satisfacer a los demás, donde el sujeto o en esta caso las sujetas madreesposas, están subyugadas por el poder que ser ejercen sobre ellas. Desde esta perspectiva, las madreesposas se convierten en "seres para los otros" (Beauvoir, 2013)

La maternidad también se refiere a una forma de poder, donde viven condiciones de opresión y existe: la expropiación de sus cuerpos y general de toda su vida para que, a partir de esta relación biológica con los hijos, se asuma obligatoriamente la maternidad (Lagarde, 2003).

En el matrimonio los hombres se pueden reproducir y manifiestan su derecho de propiedad mediante los hijos; el poder es ejercido sobre la mujer por medio de la monogamia femenina que es exigida en la sociedad, así se asegura una paternidad reconocida ante la familia y la sociedad; la paternidad es una de las instituciones básicas y está compuesto por un complejo de relaciones de funciones y personajes: el padre social, el padre simbólico, entre otros. (Lagarde, 2003). En nuestra sociedad patriarcal, el padre es la cabeza de la casa, es quien da la cara por la familia, quien aparta la economía y sobre todo la autoridad y el respeto, este poder que posee culturalmente el padre, lo hereda a los hijos y quienes lo van perpetuando a través de su apellido.

La madre es una institución histórica, clave de la reproducción de la cultura y la hegemonía, creación de los consensos del sujeto a modo de vida dominante, la maternidad convierte a las mujeres como reproductoras de la cultura, son las primeras en compartir sus conocimientos a los nuevos seres, la forma en que verán la vida y como se relacionan con los demás, depende de las madres, siendo de esto modo, una forma de reproducción de la hegemonía patriarcal en la construcción de la identidad de los sujetos, de este modo la maternidad juega un papel importante en la percepción del mundo de sus hijos.

\section{Presa}

Refiriéndose a las mujeres que se encuentran en cautiverios, y todo cautiverio implica una prisión: un conjunto de límites, materiales y subjetivos, tabúes, prohibiciones y obligaciones impuestas en la subordinación (Lagarde, 2003).

La prisión es un castigo y mediante esta se ejerce el poder, destinada para transgresores y para aquellos que no cumplen las normas.

Visto de este modo muchas mujeres viven en una prisión simbólica, donde la primera prisión es la cultura y la sociedad, seguida por el matrimonio, los hijos y los roles sociales de mujer.

\section{Loca}

En el mundo donde priva la axiología del bien y el mal, las locas son muy buenas y las muy malas, aquellas mujeres cuyo despliegue exagerado en la vida las llevo a los extremos de la sinrazón (Lagarde,2003). La locura esta encasillada como una enfermedad, que hace que no esté consciente de lo hace y de lo que dice; en los estudios de Foucault sobre la locura, argumenta que el loco carecía de validez en el discurso, por ello cuando una mujer la encasillan como loca, es para ignorar lo que dice, lo que hace e incluso tacharla de enferma.

\section{Leyes en el marco de apoyo a las mujeres en México}

Durante décadas se ha buscado garantizar los derechos de las mujeres en México, se ha ido avanzando por etapas en diferentes rubros como son: derecho al voto, derecho a asistir a la universidad, derecho a divorciarse, entre muchas otras cosas más.

Uno de los grandes pasos que se ha logrado en México es la Ley general de acceso de las mujeres a una vida libre de violencia.

En el 2007 se publicó esta ley, donde por primera vez en el país se reconoció que las mujeres no solo sufren violencia física, sino también existe la psicológica, económica, simbólica, entre otras. Esta y otras leyes han ayudado a disminuir la violencia contra las mujeres en todos los aspectos. Es importante mencionar que en esta ley se menciona la alerta de violencia de género.

A continuación, se presentan algunas leyes en apoyo a las mujeres en México.

Año Ley

2001 se creó por Ley el Instituto Nacional de las Mujeres (IMUJERES), esto en consecuencia de un trabajo de la sociedad civil y activistas en Género.

2006 fue promulgada la Ley General para la Igualdad entre Mujeres y Hombres (LGIMH), esta tiene el objetivo 
de regular y garantizar la igualdad entre ambos, así mismo como sugiere los mecanismos para lograr esa igualdad real en México.

2007 se promulgó la Ley General de Acceso a las mujeres de una vida libre Violencia, estableciendo los lineamientos jurídicos y administrativos que garantizan el derecho de las mujeres a vivir sin violencia, esta ley trabaja en coordinación de los estados y los municipios. Los estados cuentan con sus leyes que garantizan a las mujeres una vida libre de violencia.

\begin{tabular}{|c|l|}
\hline Año 2001 & \multicolumn{1}{|c|}{ Ley } \\
\hline 2006 & $\begin{array}{l}\text { Se creó por Ley el Instituto Nacional de las } \\
\text { Mujeres (IMUJERES), esto en consecuencia } \\
\text { de un trabajo de la sociedad civil y activistas } \\
\text { en Género. }\end{array}$ \\
\hline \multirow{2}{*}{$\begin{array}{l}\text { Fue promulgada la Ley General para la } \\
\text { lgualdad entre Mujeres y Hombres (LGIMH), } \\
\text { esta tiene el objetivo de regular y garantizar } \\
\text { la igualdad entre ambos, así mismo como } \\
\text { sugiere los mecanismos para lograr esa } \\
\text { igualdad real en México. }\end{array}$} \\
$\begin{array}{l}\text { Se promulgó la Ley General de Acceso a las } \\
\text { mujeres de una vida libre Violencia, } \\
\text { estableciendo los lineamientos jurídicos y } \\
\text { administrativos que garantizan el derecho de } \\
\text { las mujeres a vivir sin violencia, esta ley } \\
\text { trabaja en coordinación de los estados y los } \\
\text { municipios. Los estados cuentan con sus } \\
\text { leyes que garantizan a las mujeres una vida } \\
\text { libre de violencia. }\end{array}$ \\
\hline
\end{tabular}

Tabla 1 Leyes de apoyo a las mujeres en México. Autoría propia.

\section{Metodología e instrumento de investigación}

El tipo de estudio es de tipo descriptivo, ya que se requiere situar a las alumnas en la realidad actual, a partir de su sexo y de la circunstancia de la pandemia. Se elaboró un instrumento de acuerdo con las categorías de análisis que propone Marcela Lagarde, tomado en cuenta la edad de las alumnas, se retomaron solo las categorías analíticas: madreesposas, presa, loca. Se realizaron ítems con respecto al poder pastoral de Michael Foucault y se monitoreo con respecto a la violencia en casa.

\section{Muestra}

La fórmula para calcular el tamaño de muestra cuando se conoce el tamaño de la población es la siguiente:

$$
n=\frac{N \times Z_{a}{ }^{2} \times p \times q}{d^{2} \times(N-1)+Z_{a}{ }^{2} \times p \times q}
$$

En donde,

$\mathrm{N}$ = tamaño de la población,

$Z=$ nivel de confianza,

$\mathrm{P}=$ probabilidad de éxito o proporción esperada,

$Q=$ probabilidad de fracaso,

$\mathrm{D}=$ precisión (Error máximo admisible en términos de proporción).

$$
n=\frac{1300 * 0.95^{2}}{0.04^{2} * 1299+0.95} \approx 388
$$

\section{Resultados}

El instrumento fue cualitativo y cuantitativo con la finalidad de sistematizar argumentos y rescatar porcentajes de las alumnas; se aplicó a 388 alumnas del nivel bachillerato de la Escuela Superior de Tizayuca.

La edad de las alumnas oscila de los 15 hasta los 18 años, repartidos de la siguiente forma: el $2^{\circ} .2 \%$ de 15 años, el $31.3 \%$ de 16 años, el $36.5 \%$ de 17 años y el $12.1 \%$ de 18 o más años.

\section{La familia}

La familia es el núcleo de la sociedad, es el pilar de toda institución, mismo que reproduce la cultura y las costumbres; este ítem se aplicó con finalidad de conocer la cantidad de integrantes que conforman su familia y suponer la situación familiar que viven.

¿Cuántos integrantes conforman tu familia?

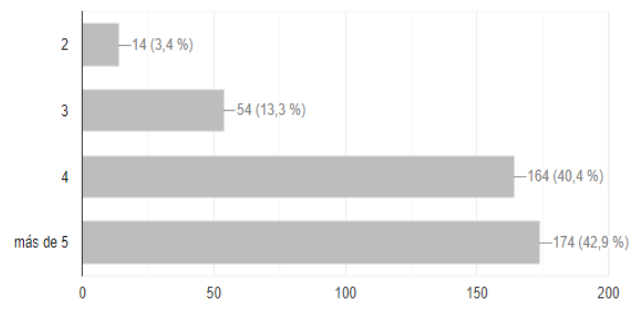

\section{Gráfico 1}

Se puede observar que un $42.9 \%$ de las familias de las alumnas, está conformada por más de cinco integrantes; el $40.4 \%$ de las familias de las alumnas está conformado por 4 integrantes y el $17.7 \%$ están integrados por 2 y 3 familiares. De acuerdo con lo anterior se supone que estas últimas alumnas han sufrido mayor impacto económico durante la pandemia, por el número de integrantes que conforman sus familias ya que, a más integrantes, mayores gastos familiares. 
¿La cabeza de tu familia es una mujer?

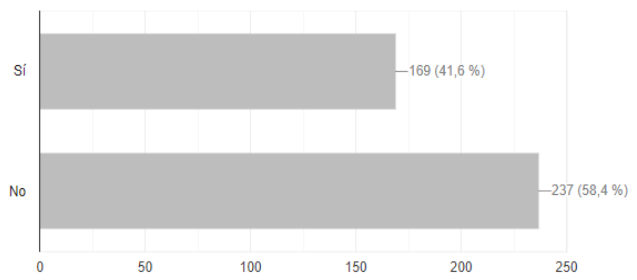

\section{Gráfico 2}

El 41.5 de las alumnas respondieron que la cabeza de su familia son mujeres, este dato es relevante porque: "Según datos de la Encuesta Nacional de Ocupación y Empleo (ENOE) para 2019, el 73\% (36.2 millones) de las mujeres de 15 años y más residentes en nuestro país, ha tenido al menos una hija o hijo nacido vivo, de ellas el $9.9 \%$ son madres solteras" (INMUJERES, 2019). Y por lo tanto se observa un alto porcentaje de mujeres como cabeza de familias en las alumnas de la ESTi.

¿Tu familia se ha visto afectada económicamente por la pandemia?

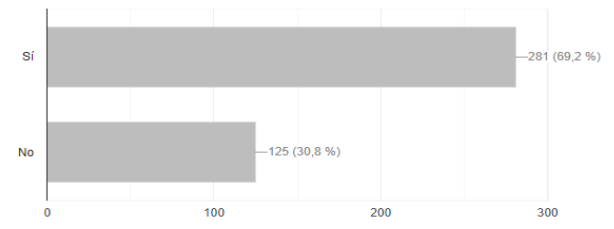

Gráfico 3

Se observa que el "69.2\% de las familias se han visto afectadas económicamente por la pandemia, según el Banco Mundial, el pronóstico de crecimiento de población en condiciones de pobreza extrema va en aumento, debido a que el COVID-19 ha ocasionado un crecimiento de desempleo y bajas expectativas en la economía mundial, los más afectados serán los que viven cerca de la línea de afectación de la pobreza extrema" (Hernández \& Mar, 2020).

¿Tienes hijos?

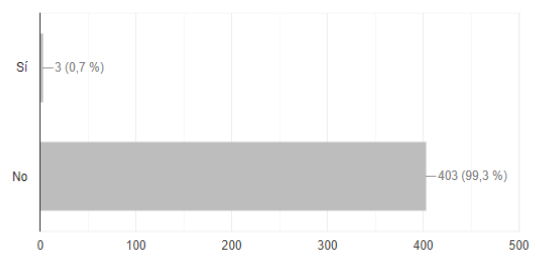

Gráfico 4

Se puede observar que solo tres alumnas de las encuestadas tienen hijos, dato de relevancia para la categoría de análisis madreesposas de Marcela Lagarde.
¿Durante el periodo de pandemia te ha tocado cuidar a tus hermanos, sobrino o algún menor de edad?

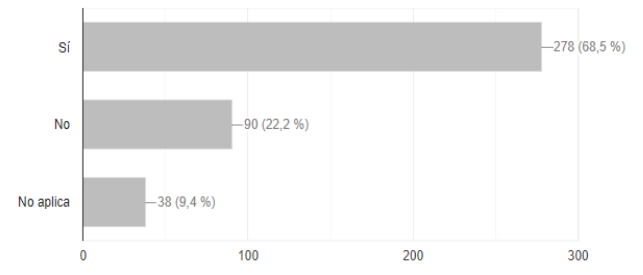

\section{Gráfico 5}

¿Te ha tocado cuidar a algún familiar enfermo durante la época de pandemia?

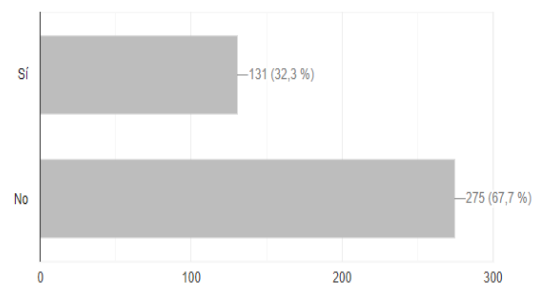

\section{Gráfico 6}

Se puede observar que el $68.5 \%$ de las alumnas han tenido que cuidar a hermanos, sobrinos, o algún menor de edad, durante el periodo de pandemia; y el $32.3 \%$ han cuidado a familiares enfermos de COVID 19. Se comprueba la teoría de Lagarde donde la mujer, de acuerdo con la cultura y sociedad, está ligada a los cuidados, aunque no sea madre (Lagarde, 2003).

¿Has sacrificado alguna actividad personal por cuidar a tu familia?

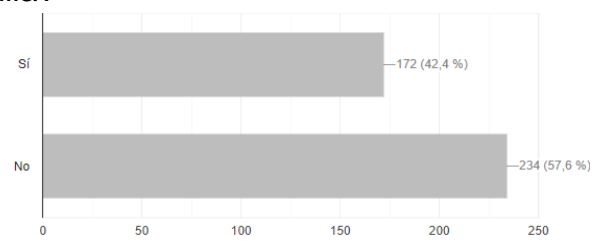

Gráfico 7

El $42.4 \%$ de las alumnas de nivel bachillerato de la ESTi consideran que han sacrificado actividades personales por estar al cuidado de sus familiares enfermos, al tanto de los quehaceres de la casa o cargo de los menores de edad de su familia.

¿Consideras que es "justo" el reparto de actividades en tu casa? 


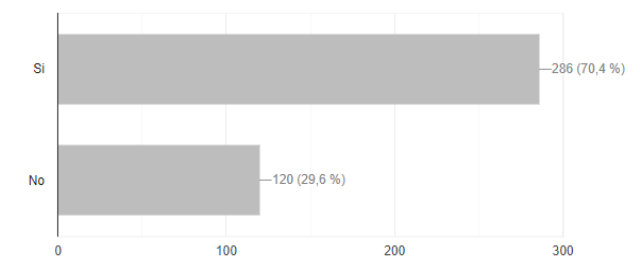

Gráfico 8

El $70.4 \%$ de las alumnas comentan que si consideran justo el reparto de actividades que realizan en su casa y solo el $29.6 \%$ consideran lo contrario. Es interesante que este último porcentaje de alumnas manifiestan que sus familiares hombres no realizan las mismas actividades que ellas, que "les cargan la mano por ser las mayores", "que las tratan como sirvientas" etc.

Durante la época de pandemia ¿Te has sentido atacada por algún familiar?

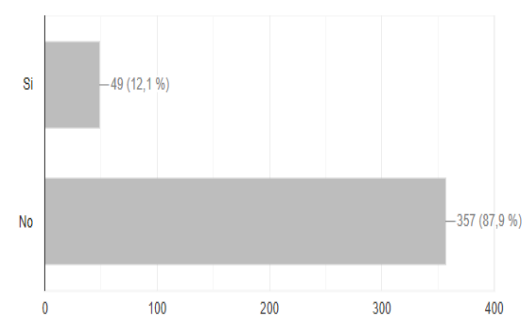

\section{Gráfico 9}

De acuerdo con el gráfico, se observa que el $12.1 \%$ de las alumnas han sufrido algún tipo de ataque por algún familiar. Es importante resaltar que las alumnas tienen derecho a una vida libre de violencia, está estipulado en la ley del Estado de Hidalgo.

\section{Conclusiones}

De acuerdo con la teoría presentada y los resultados de la encuesta se confirma que las alumnas tienen un mayor peso familiar en las actividades con respecto al cuidado y labores de la casa, asumiendo el papel de madresesposas, de la misma forma se observa que las alumnas manifiesta en un $42 \%$ que, si sacrifican actividades personales por cuidar o apoyar a su familia, asumiendo de alguna manera el cautiverio de presas.

Las alumnas del nivel bachillerato se encuentran en una etapa complicada por mucha razones, en un primer momento viven una doble o triple jornada en su mayoría, ya que apoyan en las labores de limpieza de la casa, trabajan en el exterior para apoyar la economía familiar, apoyan a sus familiares en sus trabajos que realizan desde casa, apoyan en los negocios familiares, con las tareas escolares de los menores de edad, cuidan enfermos de COVID y después de todo esto, realizan sus actividades escolares del nivel bachillerato.

El poder pastoral es una manifestación del poder, donde Michel Foucault hace referencia a un pastor con sus ovejas, donde logra que las personas interioricen ese ejercicio del poder y se sientan comprometidas y obligadas a realizar ciertas actividades, como es el caso de las alumnas, donde manifiestan de manera expresa que ellas tienen la obligación de servir solo por el hecho de ser hermanas mayores o mujeres.

La pandemia vino a destabilizar a las instituciones, se ha afectado a la economía mundial, al sector salud, pero también a la estabilidad emocional de las personas. Con los datos que arrojó el instrumento, se puede observar que las alumnas son sometidas al ejercicio de un poder pastoral y en los cautiverios que describe Largade; las mujeres siempre son afectas a lo largo de los desastres mundiales, por la desventaja histórica que se tiene, esto es se logrará cambiar con el reemplazo de idiosincrasia y compartir la nueva cultura no patriarcal.

\section{Referencias}

Beauvoir, S. d. (2013). El segundo Sexo. México: Debolsillo. CNDH. (02 de abril de 2021). Género, masculinidades y lenguaje incluyente y no sexista. Obtenido de Módulo 1. Sistema SexoGénero

Foucault, M. (1988). El sujeto y el poder. Francia.

Hernàndez, A., \& Mar, P. J. (Septiembre de 2020). Efectos de la pandemia en la econimia familiar. México, Estado de Mexico.

INMUJERES. (19 de septiembre de 2019). Instituto nacional de las Mujeres. Obtenido de

http://estadistica.inmujeres.gob.mx/formas/tarjetas/Madres_solt eras.pdf

Lagarde, M. (2003). Los cautiverios de las mujeres: Madresposas, monjas, putas, presas y locas. México: Siglo XXI Editores México.

OMS. (24 de Febrero de 2010). Organización Mundial de la Salud. Obtenido de

https://www.who.int/csr/disease/swineflu/frequently_asked_qu estions/pandemic/es/

UAEH. (16 de 03 de 2020). Obtenido de Boletín: https://www.uaeh.edu.mx/noticias/5470/ 